\title{
from Boolean Algebra to Unified Algebra
}

\author{
Eric C. R. Hehner \\ University of Toronto
}

\begin{abstract}
Boolean algebra is simpler than number algebra, with applications in programming, circuit design, law, specifications, mathematical proof, and reasoning in any domain. So why is number algebra taught in primary school and used routinely by scientists, engineers, economists, and the general public, while boolean algebra is not taught until university, and not routinely used by anyone? A large part of the answer may be in the terminology and symbols used, and in the explanations of boolean algebra found in textbooks. This paper points out some of the problems delaying the acceptance and use of boolean algebra, and suggests some solutions.
\end{abstract}

\section{Introduction}

This paper is about the symbols and notations of boolean algebra, and about the way the subject is explained. It is about education, and about putting boolean algebra into general use and practice. To make the scope clear, by "boolean algebra" I mean the algebra whose expressions are of type boolean. I mean to include the expressions of propositional calculus and predicate calculus. I shall say "boolean algebra" or "boolean calculus" interchangeably, and call the expressions of this algebra "boolean expressions". Analogously, I say "number algebra" or "number calculus" interchangeably, and call the expressions of that algebra "number expressions".

Boolean algebra is the basic algebra for much of computer science. Other applications include digital circuit design, law, reasoning about any subject, and any kind of specifications, as well as providing a foundation for all of mathematics. Boolean algebra is inherently simpler than number algebra. There are only two boolean values and a few boolean operators, and they can be explained by a small table. There are infinitely many number values and number operators, and even the simplest, counting, is inductively defined. So why is number algebra taught in primary school, and boolean algebra in university? Why isn't boolean algebra better known, better accepted, and better used?

One reason may be that, although boolean algebra is just as useful as number algebra, it isn't as necessary. Informal methods of reckoning quantity became intolerable several thousand years ago, but we still get along with informal methods of specification, design, and reasoning. Another reason may be just an accident of educational history, and still another may be our continuing mistreatment of boolean algebra.

\section{Historical Perspective}

To start to answer these questions, I'm going to look briefly at the history of number algebra. Long after the invention of numbers and arithmetic, quantitative reasoning was still a matter of trial and error, and still conducted in natural language. If a man died leaving his 3 goats and 20 chickens to be divided equally between his 2 sons, and it was agreed that a goat is worth 8 
chickens, the solution was determined by iterative approximations, probably using the goats and chickens themselves in the calculation. The arithmetic needed for verification was well understood long before the algebra needed to find a solution.

The advent of algebra provided a more effective way of finding solutions to such problems, but it was a difficult step up in abstraction. The step from constants to variables is as large as the step from chickens to numbers. In English 500 years ago, constants were called "nombers denominate" [concrete numbers], and variables were called "nombers abstracte". One of the simplest, most general laws, sometimes called "substitution of equals for equals", $x=y \Rightarrow f x=f y$

seems to have been discovered a little at a time. Here is one special case [20]:

“IJn the firste there appeareth 2 nombers, that is $14 x+15 y$ equalle to one nomber, whiche is $7 \mathfrak{l y}$. Thut if you marke them well, you maie see ome denomination, on bothe sidos of the equation, which never ought to stand. Whyerfore abating [subtracting] the lesser, that is $15 \mathrm{y}$ out of bothe the nombers, there will remain $14 x=56$ y that $i s$, by reduction, $1 x=4 y$.

Sibolar. IJ see, pou abate $15 y$ from them bothe. And then are thei equalle still, sepng thei wer equalle before. According to the thirde common sentence, in the patthemaie: Jff pou abate enen [equal] portions, from thynges that bee equalle, the partes that remain shall be equall also. Atlaster. 翼ou doe well remember the firste arounds of this arte."

And then, a paragraph later, another special case:

"Ilf you adde equalle portions, to thpnges that bee equalle, what so amounteth of them shall be equalle."

Each step in an abstract calculation was accompanied by a concrete justification. For example, we have the Commutative Law [0]:

Z9hen the chekpns of two gentle menne are counted, we may count first the thekpns of the aentplman having fewer chekpns, and after the thekpns of the gentplman having the areater portion. Jlf the nomber of the greater portion be counted first, and then that of the lesser portion, the denomination so determined shall be the same.

This version of the Commutative Law includes an unnecessary case analysis, and it has missed a case: when the two gentlemen have the same number of chickens, it does not say whether the order matters. The Associative Law [0]:

29hen thenges to be counted are divided in two partes, and lately are found moare thenges to be counted in the same aemerall quantitie, it matters not whether the thpnges lately added be counted together with the lesser parte or with the areater parte, or that there are severalle partes and the thynges lately added be counted together with any one of them.

As you can imagine, the distance from $2 x+3=3 x+2$ to $x=1$ was likely to be several pages. The reason for all the discussion in between formulas was that algebra was not yet fully trusted. Algebra replaces meaning with symbol manipulation; the loss of meaning is not easy to accept. The author constantly had to reassure those readers who had not yet freed themselves from thinking about the objects represented by numbers and variables. Those who were skilled in the art of informal reasoning about quantity were convinced that thinking about the objects helps to calculate correctly, because that is how they did it. As with any technological advance, those who are most skilled in the old way are the most reluctant to see it replaced by the new.

Today, of course, we expect a quantitative calculation to be conducted entirely in algebra, without reference to thynges. Although we justify each step in a calculation by reference to an algebraic law, we do not have to justify the laws continually. We can go farther, faster, more 
succinctly, and with much greater certainty. In a typical modern proof (see the first Appendix) we see lines like

$$
\begin{aligned}
& \lambda^{r} a^{r}=\left(b a b^{-1}\right)^{r}=b a^{r} b^{-1}=a^{r} \\
& b^{r}=\lambda^{r} b^{r}=(\lambda b)^{r}=\left(a^{-1} b a\right)^{r}=a^{-1} b^{r} a \\
& \left(a_{1}^{-1} b_{1}\right)^{2}=a_{1}^{-1} b_{1} a_{1}^{-1} b_{1}=a_{1}^{-1}\left(b_{1} a_{1}^{-1}\right) b_{1}=a_{1}^{-1}\left(\mu a_{1}^{-1} b_{1}\right) b_{1}=\mu a_{1}^{-2} b_{1}^{2} \\
& \left(a_{1}^{-1} b_{1}\right)^{r}=\mu^{1+2+\ldots+(r-1)} a_{1}^{-r} b_{1}^{r}=\mu^{1+2+\ldots+(r-1)}=\mu^{r(r-1) / 2}
\end{aligned}
$$

These lines were taken from a proof of Wedderburn's Theorem (a finite division ring is a commutative field) in [15] (the text used when I studied algebra). Before we start to feel pleased with ourselves at the improvement, let me point out that there is another kind of calculation, a boolean calculation, occurring in the English text between the formulas. In the example proof [15] we find the words "consequently", "implying", "there is/are", "however", "thus", "hence", "since", "forces", "if...then", "in consequence of which", "from which we get", "whence", "would imply", "contrary to", "so that", "contradicting"; all these words suggest boolean operators. We also find bookkeeping sentences like "We first remark ...", "We must now rule out the case ..."; these suggest the structure of a boolean expression. It will be quite a large expression, perhaps taking an entire page. If written in the usual unformatted fashion of proofs in current algebra texts, it will be quite unreadable. The same problem occurs with computer programs, which can be thousands of pages long; to make them readable they must be carefully formatted, with indentation to indicate structure. We will have to do likewise with proofs.

A formal proof is a boolean calculation using boolean algebra; when we learn to use it well, it will enable us to go farther, faster, more succinctly, and with much greater certainty. But there is a great resistance in the mathematical community to formal proof, especially from those who are most expert at informal proof. They complain that formal proof loses meaning, replacing it with symbol manipulation. The current state of boolean algebra, not as an object of study but as a tool for use, is very much the same as number algebra was 5 centuries ago.

\section{Boolean Calculation}

Given an expression, it is often useful to find an equivalent but simpler expression. For example, in number algebra

$$
\begin{array}{ll} 
& x \times(z+1)-y \times(z-1)-z \times(x-y) \\
= & \\
= & x \times z+x \times 1)-(y \times z-y \times 1)-(z \times x-z \times y) \\
= & x+y+(x \times z-x \times z)+(y \times z-y \times z) \\
= & x+y
\end{array}
$$

distribute unity and double negation symmetry and associativity zero and identity

We might sometimes want to find an equivalent expression that isn't simpler; to remove the directionality I'll say "calculation" rather than "simplification". We can use operators other than = down the left side of the calculation; we can even use a mixture of operators, as long as there is transitivity. For example, the calculation (for real $x$ )

$$
\begin{array}{ll} 
& x \times(x+2) \\
= & x^{2}+2 \times x \\
= & x^{2}+2 \times x+1-1 \\
= & (x+1)^{2}-1 \\
\geq & -1
\end{array}
$$

tells us

$$
x \times(x+2) \geq-1
$$

add and subtract 1

factor

a square is nonnegative 
Boolean calculation is similar. For example,

$$
\begin{array}{ll} 
& (a \Rightarrow b) \vee(b \Rightarrow a) \\
\equiv & \neg a \vee b \vee \neg b \vee a \\
\equiv & a \vee \neg a \vee b \vee \neg b \\
\equiv & \text { true } \vee \text { true } \\
\equiv & \text { true }
\end{array}
$$

replace implications

$\mathrm{v}$ is symmetric excluded middle, twice $\mathrm{v}$ is idempotent

And so $(a \Rightarrow b) \vee(b \Rightarrow a)$ has been simplified to true, which is to say it has been proven. Here is another example.

$$
\begin{array}{ll} 
& \exists n \cdot n+n^{2}=n^{3} \\
\Leftarrow & 0+0^{2}=0^{3} \\
\equiv & \text { true }
\end{array}
$$

instance arithmetic

And so $\left(\exists n \cdot n+n^{2}=n^{3}\right) \Leftarrow$ true , and so $\exists n \cdot n+n^{2}=n^{3}$ is proven.

Solving simultaneous equations can also be done as a boolean calculation. For example,

$$
\begin{array}{llr} 
& x+x \times y+y=5 \wedge x-x \times y+y=1 & \text { subtract and add } 2 \times x \times y \text { in first equation } \\
\equiv & x-x \times y+y+2 \times x \times y=5 \wedge x-x \times y+y=1 & \text { use second equation to simplify first } \\
\equiv & 1+2 \times x \times y=5 \wedge x-x \times y+y=1 & \\
\equiv & 2 \times x \times y=4 \wedge x-x \times y+y=1 & \\
\equiv & x \times y=2 \wedge x-x \times y+y=1 & \\
\equiv & x \times y=2 \wedge x-2+y=1 & \\
\equiv & x \times y=2 \wedge x+y=3 & \\
\equiv & x=1 \wedge y=2 \vee x=2 \wedge y=1 & \\
\Leftarrow & x=1 \wedge y=2
\end{array}
$$

These examples show that simplifying, proving, and solving are all the same: they are all just calculation.

When an expression is too long to fit on one line, it must be nicely formatted for easy reading, and when a hint is too long to fit on the remainder of a line, it can be written on as many lines as it takes, but I do not consider formatting further here. One point worth mentioning is that subcalculations (if boolean, they are called subproofs or lemmas) can save copying unchanged parts of a calculation through many lines. These subcalculations can be done in another place and referenced, or they can be done in-place, nicely formatted, to provide a structured calculation (structured proof). By far the best way to handle subcalculations is provided by window inference systems [21] [2], which open a new window for each subcalculation, keep track of its sense (direction), and make its context available. For example, in solving the simultaneous equations, I used the second equation to simplify the first, and then the first to simplify the second.

In this brief introduction to boolean calculation, I have not taken the time to present all the rules. For a complete presentation, the reader is referred to [14]. A research monograph that uses calculational proof is [7]. A textbook on discrete math that uses calculational proof is [10]. For further discussion of calculational proofs see [9] [17].

\section{Traditional Terminology}

Formal logic has developed a complicated terminology that its students are forced to learn. There are terms, which are said to have values. There are formulas, also known as propositions or sentences, which are said not to have values, but instead to be true or false. Operators $(+,-)$ 
join terms, while connectives $(\Lambda, \vee)$ join formulas. Some terms are boolean, and they have the value true or false, but that's different from being true or false. It is difficult to find a definition of predicate, but it seems that a boolean term like $x=y$ stops being a boolean term and mysteriously starts being a predicate when we admit the possibility of using quantifiers $(\exists, \forall)$. Does $x+y$ stop being a number term if we admit the possibility of using summation and product $(\Sigma, \Pi)$ ? There are at least three different equal signs: $=$ for terms, and $\Leftrightarrow$ and $\equiv$ for formulas and predicates, with one of them carrying an implicit universal quantification. We can even find a peculiar mixture in some textbooks, such as the following:

$$
a+b=a \vee a+b=b
$$

Here, $a$ and $b$ are boolean variables, + is a boolean operator (disjunction), $a+b$ is a boolean term (having value true or false), $a+b=a$ and $a+b=b$ are formulas (so they are true or false), and finally $v$ is a logical connective.

Fortunately, in the past few decades there has been a noticeable shift toward erasing the distinction between being true or false and having the value true or false. It is a shift toward the calculational style of proof. But we have a long way to go yet, as I find whenever I ask my beginning students to prove something of the form $a \oplus b$ where $\oplus$ is pronounced "exclusive or". They cannot even start because they expect something that looks grammatically like a sentence. If I change it to either of the equivalent forms $(a \oplus b) \equiv$ true or $a \neq b$ they are happy because they can read it as a sentence with a verb. But $(a \neq b) \equiv$ true confuses them again because it seems to have too many verbs. If I ask them to prove something of the form $a v b$, they take an unwittingly constructivist interpretation, and suppose I want them to prove $a$ or prove $b$ because that is what "do $a$ or $b$ " means in English. The same lack of understanding can be found in many introductory programming texts where boolean expressions are not taught in their generality but as comparisons because comparisons have verbs. We find

while flag=true do something od

but not the equivalent, simpler, more efficient

while flag do something od

because flag isn't the right part of speech to follow while . Our dependence on natural language for the understanding of boolean expressions is a serious impediment.

\section{Traditional Notations}

Arithmetic notations are reasonably standard throughout the world. The expression $738+45=783$

is recognized and understood by schoolchildren almost everywhere. But there are no standard boolean notations. Even the two boolean constants have no standard symbols. Symbols in use include

$\begin{array}{lllll}\text { true } & \mathrm{t} & \mathrm{T} & 1 & 0 \\ \text { false } & \mathrm{f} & \mathrm{F} & 0 & 1\end{array}$

Quite often the boolean constants are written as 1 and 0 , with + for disjunction, adjacency for conjunction, and perhaps - for negation. With this notation, here are some laws.

$$
\begin{aligned}
& x(y+z)=x y+x z \\
& x+y z=(x+y)(x+z) \\
& x+-x=1 \\
& x(-x)=0
\end{aligned}
$$

The first law above coincides with number algebra, but the next three clash with number algebra. The overwhelming reaction of algebraists to notational criticisms is: it doesn't matter which symbols are used; just introduce them, and get on with it. But to apply an algebra, one must 
recognize the patterns, matching laws to the expression at hand. The laws have to be familiar. It takes an extra moment to think which algebra I am using as I apply a law. The logician R.L. Goodstein [8] chose to use 0 and 1 the other way around, which slows me down a little more. A big change, like using + as a variable and $x$ as an operator, would slow me down a lot. I think it matters even to algebraists because they too have to recognize patterns. To a larger public, the reuse of arithmetic symbols with different meanings is an insurmountable obstacle. And when we mix arithmetic and boolean operators in one expression, as we often do, it is impossible to disambiguate.

The most common notations for the two boolean constants found in programming languages and in programming textbooks seem to be true and false. I have two objections to these symbols. The first is that they are English-based and clumsy. Number algebra could never have advanced to its present state if we had to write out words for numbers.

seven three eight + four five $=$ seven eight three

is just too clumsy, and so is

true $\wedge$ false $\vee$ true $\equiv$ true

Clumsiness may seem minor, but it can be the difference between success and failure in a calculus.

My second, and more serious, objection is that the words true and false confuse the algebra with an application. One of the primary applications of boolean algebra is to formalize reasoning, to determine the truth or falsity of some statements from the truth or falsity of others. In that application, we use one of the boolean constants to represent truth, and the other to represent falsity. So for that application, it seems reasonable to call them true and false. The algebra arose from that application, and it is so much identified with it that many people cannot separate them; they think the boolean values really are true and false. But of course boolean expressions are useful for describing anything that comes in two kinds. We apply boolean algebra to circuits in which there are two voltages. We sometimes say that there are $0 \mathrm{~s}$ and $1 \mathrm{~s}$ in a computer's memory, or that there are trues and falses. Of course that's nonsense; there are neither $0 \mathrm{~s}$ and $1 \mathrm{~s}$ nor trues and falses in there; there are low and high voltages. We need symbols that can represent truth values and voltages equally well.

Boolean expressions have other applications, and the notations we choose should be equally appropriate for all of them. Computer programs are written to make computers work in some desired way. Before writing a program, a programmer should know which ways are desirable and which are not. That divides computer behavior into two kinds, and we can use boolean expressions to represent them. A boolean expression used this way is called a specification. We can specify anything, not just computer behavior, using boolean expressions. For example, if you would like to buy a table, then tables are of two kinds: those you find desirable and are willing to buy, and those you find undesirable and are not willing to buy. So you can use a boolean expression as a table specification. Acceptable and unacceptable human behavior is specified by laws, and boolean expressions have been proposed as a better way than legal language for writing laws [1]. They can be used to calculate the attractions and repulsions among a set of magnets.

For symbols that are independent of the application, I propose the lattice symbols $T$ and $\perp$, pronounced "top" and "bottom". Since boolean algebra is the mother of all lattices, I think it is appropriate, not a misuse of those symbols. They can equally well be used for true and false statements, for high and low voltages (power and ground), for satisfactory and unsatisfactory 
tables, for innocent and guilty behavior, or any other opposites.

For disjunction, the symbol $\vee$ is fairly standard, coming from the Latin word "vel" for "or". For conjunction, the symbol is less standard, the two most common choices being \& and $\wedge$. We are even less settled on a symbol for implication. Symbols in use include

$$
\rightarrow \Rightarrow \therefore \supset
$$

The usual explanation says it means "if then", followed by a discussion about the meaning of "if then". Apparently, people find it difficult to understand an implication whose antecedent is false ; for example, "If my mother had been a man, I'd be the king of France." [19]. Such an implication is called "counter-factual". Some people are uneasy with the idea that false implies anything, so some researchers in Artificial Intelligence have proposed a new definition of implication. The following truth table shows both the old and new definitions.

\begin{tabular}{cccc}
$a$ & $b$ & $\begin{array}{c}\text { old } \\
a \Rightarrow b\end{array}$ & $\begin{array}{c}\text { new } \\
a \Rightarrow b\end{array}$ \\
\hline true & true & true & true \\
true false & false & false \\
false & true & true & unknown \\
false false & true & unknown
\end{tabular}

where unknown is a third boolean value. When the antecedent is false, the result of the new kind of implication is unknown. This is argued to be more intuitive. I believe this proposal betrays a serious misunderstanding of logic. When someone makes a statement, they are saying that the statement is true. Even if the statement is "if $a$ then $b$ " and $a$ is known to be false, nonetheless we are being told that "if $a$ then $b$ " is true. It is the consequent $b$ that is unknown. And that is represented perfectly by the old implication: there are two rows in which $a$ is false and $a \Rightarrow b$ is true; on one of these rows, $b$ is true, and on the other $b$ is false.

Debate about implication has been going on for a long time; 22 centuries ago, Callimachus, the librarian at Alexandria, said "Even the crows on the roof croak about what implications are sound."[3] [18]. In case you think that confusion is past, or just for beginners, consider the explanation of implication in Contemporary Logic Design, 1994 [16]:

"As an example, let's look at the following logic statement:

IF the garage door is open

AND the car is running

THEN the car can be backed out of the garage

It states that the conditions - the garage is open and the car is running - must be true before the car can be backed out. If either or both are false, then the car cannot be backed out."

Even a Berkeley computer science and electrical engineering professor can get implication wrong.

Implication is best presented as an ordering. If we are calling the boolean values "top" and "bottom", we can say "lower than or equal to" for implication. It is easy, even for primary school students, to accept that $\perp$ is lower than or equal to $T$, and that $\perp$ is lower than or equal to $\perp$. With this new pronunciation and explanation, three other neglected boolean operators become familiar and usable; they are "higher than or equal to", "lower than", and "higher than". For lack of a name and symbol, the last two operators have been treated like shameful secrets, and shunned. If we are still calling the boolean values "true" and "false", then we shall have to call implication "falser than or equal to". As we get into boolean expressions 
that use other types, ordering remains a good explanation: $x<4$ is falser than or equal to $x<6$, as a sampling of evaluations illustrates (try $x=3,5,7$ ). I have tried using the standard words "stronger" and "weaker", saying $x<4$ is stronger than $x<6$; but I find that some of my students have an ethical objection to saying that falsity is stronger than truth.

That implication is the boolean ordering, with $T$ and $\perp$ at the extremes, is not appreciated by all who use boolean algebra. In the specification language $Z$ [24], boolean expressions are used as specifications. Specification $A$ refines specification $B$ if all behavior satisfying $A$ also satisfies $B$. Although increasing satisfaction is exactly the implication ordering, the designers of $\mathrm{Z}$ defined a different ordering for refinement where $T$ is not satisfied by all computations, only by terminating computations, and $\perp$ is satisfied by some computations, namely nonterminating computations. They chose to embed a new lattice within boolean algebra, rather than to use the lattice that it provides.

Implication has often been defined as a "secondary" operator in terms of the "primary" operators negation and disjunction:

$$
(a \Rightarrow b) \equiv \neg a \vee b
$$

Proofs about implications proceed by getting rid of them in favor of the more familiar negation and disjunction, as I did earlier in an example. This avoids the informal explanation, but it makes an unsupportable distinction between "primary" and "secondary" operators, and hides the fact that it is an ordering. When we learn that implication is an ordering, proofs about implications become shorter and easier.

If we present implication as an ordering, as I prefer, then we face the problem of how to use this ordering in the formalization of natural language reasoning. To what extent does the algebraic operator "lower than or equal to" correspond to the English word "implication"? Philosophers and linguists are welcome to consider this question. But we shouldn't let the complexities of this application of boolean algebra complicate the algebra, any more than we let the complexities of the banking industry complicate the definition of arithmetic.

\section{Symmetry and Duality}

In choosing infix symbols, there is a simple principle that really helps our ability to calculate: we should choose symmetric symbols for symmetric operators, and asymmetric symbols for asymmetric operators, and choose the reverse of an asymmetric symbol for the reverse operator. The benefit is that a lot of laws become visual: we can write an expression backwards and get an equivalent expression. For example, $x+y<z$ is equivalent to $z>y+x$. By this principle, the arithmetic symbols $+x<>=$ are well chosen but - and $\neq$ are not. The boolean symbols $\wedge$ $\vee \Rightarrow \Leftarrow \equiv \oplus$ are well chosen, but $\not \equiv$ is not.

Duality can be put to use, just like symmetry, if we use vertically symmetric symbols for selfdual operators, and vertically asymmetric symbols for non-self-dual operators with the vertical reverse for their duals. The laws that become visual are: to negate an expression, turn it upside down. For example, $(T \wedge-\perp) \vee \perp$ is the negation of $(\perp \vee-T) \wedge T$ if you allow me to use the vertically symmetric symbol - for negation, which is self-dual. There are two points that require attention when using this rule. One is that parentheses may need to be added to maintain the precedence; but if we give dual operators the same precedence, there's no problem. The other point is that variables cannot be flipped, so we negate them instead (since flipping is equivalent to negation). The well-known example is deMorgan's law: to negate $a \vee b$, turn it 
upside down and negate the variables to get $-a \wedge-b$. By this principle, the symbols $\top \perp-\wedge$ $\checkmark$ are well chosen, but $\Rightarrow \Leftarrow \equiv \not \equiv$ are not. By choosing better symbols we can let the symbols do some of the work of calculation, moving it to the level of visual processing.

\section{from Booleans to Numbers}

Some boolean expressions are laws: they have value $T$ no matter what values are assigned to the variables. Some boolean expressions are unsatisfiable: they have value $\perp$ no matter what values are assigned to the variables. The remaining boolean expressions are in between, and "solving" means finding an assignment of values for the variables for which the boolean expression has value $T$. (Solving is not just for equations but for any kind of boolean expression.) A lot of mathematics is concerned with solving. And in particular, number algebra has developed by the desire to solve. To caricature the development, we choose an unsatisfiable boolean expression and say "What a pity that it has no solutions. Let's give it one.". This has resulted in an increasing sequence of domains, from naturals to integers to rationals to reals to complex numbers. The boolean expression $x+1=0$ is unsatisfiable in the natural numbers, but we give it a solution and thereby invent the integers. Similarly we choose to give solutions to $x \times 2=1, x^{2}=2, x^{2}=-1$, and thereby progress to larger domains. This progression is both historical and pedagogical. At the same time as we gain solutions, we lose laws, since the laws and unsatisfiable expressions are each other's negations. For example, when we gain a solution to $x^{2}=2$, we lose the law $x^{2} \neq 2$.

As the domain of an operator or function grows, we do not change its symbol; addition is still denoted + as we go from naturals to complex numbers. I will not argue whether the naturals are a subset of the complex numbers or just isomorphic to a subset; for me the question has no meaning. But I do argue that it is important to use the same notation for natural 1 and complex 1 because they behave the same way, and for natural + and complex + because they behave the same way on their common domain. To be more precise, all boolean expressions over the naturals retain the same solutions over the complex numbers, and all laws of complex arithmetic that can be interpreted over the naturals are laws of natural arithmetic. The reason we must use the same symbols is so that we do not have to relearn all the solutions and laws as we enlarge or shrink the domain. And indeed, it is standard mathematical practice to use the same symbols.

For exactly the same good reasons that we have a unified treatment of number algebras, we must now unify boolean and number algebras. The question whether boolean is a different type from number is no more relevant than the question whether natural and integer are different types. What's important is that solutions and laws are learned once, in a unified system, not twice in conflicting systems. And that matters both to primary school students who must struggle to learn what will be useful to them, and to professional mathematicians who must solve and apply laws. Historically, number algebra did not grow from boolean algebra; but pedagogically it can do so. As already argued, the use of $01+\times$ for $\perp T \vee \wedge$ doesn't work. To find an association between booleans and numbers that works for unification, we must use a number system extended with an infinite number. Such a system is useful for many purposes; for example, it is used in [13] to prove things about the execution time of programs (some execution times are infinite). For a list of axioms of this arithmetic, please see [13] [14]. The association that works is as follows. 


\begin{tabular}{rccl}
\multicolumn{2}{c}{ boolean } & \multicolumn{2}{c}{ number } \\
top & $\top$ & $\infty$ & infinity \\
bottom & $\perp$ & $-\infty$ & minus infinity \\
negation & $\neg$ & - & negation \\
conjunction & $\wedge$ & $\downarrow$ & minimum \\
disjunction & $\vee$ & $\uparrow$ & maximum \\
implication & $\Rightarrow$ & $\leq$ & order \\
equivalence & $\equiv$ & $=$ & equality \\
exclusive or & $\oplus$ & $\neq$ & inequality
\end{tabular}

With this association, all number laws employing only these operators correspond to boolean laws. For example,

$\begin{array}{ll}\frac{\text { boolean law }}{\top \oplus \perp} & \frac{\text { number law }}{\infty \neq-\infty} \\ a \equiv \neg \neg a & x=--x \\ a \vee \top \equiv \top & x \uparrow \infty=\infty \\ a \wedge \perp \equiv \perp & x \downarrow-\infty=-\infty \\ a \vee \perp \equiv a & x \uparrow-\infty=x \\ a \wedge \top \equiv a & x \downarrow \infty=x \\ a \Rightarrow \top & x \leq \infty \\ \perp \Rightarrow a & -\infty \leq x \\ a \vee(b \wedge c) \equiv(a \vee b) \wedge(a \vee c) & x \uparrow(y \downarrow z)=(x \uparrow y) \downarrow(x \uparrow z) \\ a \wedge(b \vee c) \equiv(a \wedge b) \vee(a \wedge c) & x \downarrow(y \uparrow z)=(x \downarrow y) \uparrow(x \downarrow z) \\ a \vee b \equiv \neg(\neg a \wedge \neg b) & x \uparrow y=-(-x \downarrow-y) \\ a \wedge b \equiv \neg(\neg a \vee \neg b) & x \downarrow y=-(-x \uparrow-y)\end{array}$

There are boolean laws that do not correspond to number laws, just as there are integer laws that are not real laws. That's another way of saying that there are unsatisfiable boolean expressions that correspond to satisfiable number expressions. We will use this for our unified development.

\section{Unified Algebra}

Here is my proposal for the symbols of a unified algebra.

$\begin{array}{rcl}\text { top } & \text { unified } & \\ \text { bottom } & \perp & \text { infinity } \\ \text { negation } & \perp & \text { minus infinity } \\ \text { conjunction } & \downarrow & \text { negation } \\ \text { disjunction } & \uparrow & \text { minimum } \\ \text { "nand" } & \downarrow & \text { negation of minimum } \\ \text { "nor" } & \uparrow & \text { negation of maximum } \\ \text { implication } & \leq & \text { order } \\ \text { reverse implication } & \geq & \text { reverse order } \\ \text { strict implication } & < & \text { strict order } \\ \text { strict reverse implication } & > & \text { strict reverse order } \\ \text { equivalence } & = & \text { equality } \\ \text { exclusive or } & \neq & \text { inequality }\end{array}$

The symbols $-\leq \geq<>=$ are world-wide standards, used by school children in all countries, 
so I dare not suggest any change to them. The symbol $\neq$ for inequality is the next best known, but I have dared to stand up the slash so that all symmetric operators have symmetric symbols and all asymmetric operators have asymmetric symbols. (Although it was not a consideration, $\neq$ also looks more like $\oplus$.) Duality has been sacrificed to standards; the pair $\leq<$ are duals, so they ought to be vertical reflections of each other; similarly the pair $\geq>$, and also $=\neq$; addition and subtraction are self-dual, and happily + and - are vertically symmetric; multiplication is not self-dual, but $x$ is unfortunately vertically symmetric.

Having unified the symbols, I suppose we should also unify the terminology. I vote for the number terminology in the right column, except that I prefer to call $T$ and $\perp$ "top" and "bottom".

The association between booleans and numbers suggested here allows the greatest number of boolean laws to be generalized to all numbers. For example, if $a, b$, and $c$ are boolean, then it is usual to define if $a$ then $b$ else $c$ fi by the law (in standard boolean notation):

if $a$ then $b$ else $c$ fi $\equiv(a \wedge b) \vee(\neg a \wedge c)$

If $a$ remains boolean but $b$ and $c$ are numbers, the if-expression on the left is still sensible (the Algol if), and this is still a law. In the unified notation it is

if $a$ then $b$ else $c$ fi $=(a \downarrow b) \uparrow(-a \downarrow c)$

The next examples, written in boolean notation, are the laws

$$
\begin{aligned}
& (a \wedge b \Rightarrow c) \equiv(a \Rightarrow c) \vee(b \Rightarrow c) \\
& (a \vee b \Rightarrow c) \equiv(a \Rightarrow c) \wedge(b \Rightarrow c)
\end{aligned}
$$

A common error is to use conjunction twice, or disjunction twice. The boolean reading " $a$ and $b$ implies $c$ if and only if $a$ implies $c$ or $b$ implies $c$ " sounds no more reasonable than " $a$ and $b$ implies $c$ if and only if $a$ implies $c$ and $b$ implies $c$ ". In unified notation,

$$
\begin{aligned}
& (a \downarrow b \leq c)=(a \leq c) \uparrow(b \leq c) \\
& (a \uparrow b \leq c)=(a \leq c) \downarrow(b \leq c)
\end{aligned}
$$

it is obvious that the minimum of $a$ and $b$ is less than or equal to $c$ when at least one of $a$ or $b$ is less than or equal to $c$, and the maximum of $a$ and $b$ is less than or equal to $c$ when both $a$ and $b$ are less than or equal to $c$. They are laws for all numbers, not just the booleans.

The arithmetic expression $x-y$ varies directly with $x$ and inversely with $y$. Thus if we increase $x$, we increase $x-y$, and if we decrease $y$ we increase $x-y$. We calculate:

$$
\begin{array}{lll} 
& x-y & \text { increase } x \text { to } x+1 \text { and so increase the whole expression } \\
\leq & (x+1)-y & \text { decrease } y \text { to } y-1 \text { and so increase the whole expression }
\end{array}
$$

Similarly the boolean expression $x \geq y$ varies directly with $x$ and inversely with $y$ (no matter whether $x$ and $y$ are numbers and $\geq$ is number comparison, or $x$ and $y$ are boolean and $\geq$ is reverse implication, or $x$ and $y$ are a mixture of number and boolean). We calculate as follows:

$$
\begin{array}{lll} 
& x \geq y & \text { increase } x \text { to } x+1 \text { and so increase the whole expression } \\
\leq & (x+1) \geq y & \text { decrease } y \text { to } y-1 \text { and so increase the whole expression }
\end{array}
$$

It is exactly the same calculation. By unifying number algebra with boolean algebra we carry our ability to calculate over from numbers to booleans. 


\section{Unified Development}

Suppose we start with boolean algebra in the unified notation, with the terminology "top", "bottom", "minimum", "maximum", "less than", and so on. Now we say: what a pity that $x=-x$ has no solution; let's give it one. The new solution is denoted 0 . While gaining a solution to some boolean expressions, we have lost some laws such as the law of the excluded middle $x \uparrow-x$.

Now we have an algebra of three values: $\top, 0, \perp$. In one application they can be used to represent "yes", "maybe", and "no"; in another they can be used to represent "large", "medium", and "small". This algebra has 27 one-operand operators, one of which is - , defined as

\begin{tabular}{c|ccc}
$x$ & $\top$ & 0 & $\perp$ \\
\hline$-x$ & $\perp$ & 0 & $\top$
\end{tabular}

It has 19683 two-operand operators, four of which are:

\begin{tabular}{c|ccccccccc}
$x y$ & $\top \top$ & $\top 0$ & $\top \perp$ & $0 \top$ & 00 & $0 \perp$ & $\perp \top$ & $\perp 0$ & $\perp \perp$ \\
\hline$x=y$ & $\top$ & $\perp$ & $\perp$ & $\perp$ & $\top$ & $\perp$ & $\perp$ & $\perp$ & $\top$ \\
$x \leq y$ & $\top$ & $\perp$ & $\perp$ & $\top$ & $\top$ & $\perp$ & $\top$ & $\top$ & $\top$ \\
$x \lll y$ & $\top$ & 0 & $\perp$ & $\top$ & 0 & 0 & 0 & 0 & 0 \\
$x \oplus y$ & $\perp$ & $\top$ & 0 & $\top$ & 0 & $\perp$ & 0 & $\perp$ & $\top$
\end{tabular}

Whether $\leq$ or $\lll$ or another operator represents implication in the presence of uncertainty can be debated, but the algebra is not affected by the debate. The operator $\oplus$ is modular (or circular) addition, and the other operators of modular arithmetic can be given similarly.

We might continue our development with a four-valued algebra and five-valued algebra, but at this point $I$ recommend filling in the space between $T$ and 0 , and between 0 and $\perp$, with all the integers. And then we progress to the rationals, the reals, and the complex numbers as usual.

The argument in favor of this unification of boolean algebra and number algebra is just as strong as the argument in favor of using the same notations for the different number algebras. But the latter is familiar, and so it seems right, while the former is unfamiliar, and for that reason alone it may seem wrong. Ultimately, the benefits will outweigh the unfamiliarity. For example, the data structure known as AND-OR trees and the algorithm that uses them become the same as the data structure and algorithm known as minimax methods; they should not have to be learned twice.

A different unification of boolean algebra and number algebra that aims at the same goal (using the same calculations for booleans and numbers), but emphasizes traditional modular arithmetic along the way, can be found in [5], a provocative work of grand scope. 


\section{from Informal to Formal}

Many mathematical notations began their life as abbreviations for some words. For example, = was introduced in [20] to mean "is equal to" (see the second Appendix):

"And to awoide the tediouse repetition of these woordes "is equalle to" $\mathfrak{I}$ will sette as $\mathfrak{I}$ doe often in woorke use, a paire of paralleles or Germowe [twin] limes of one lengthe, thus: = , because noe 2 thymages, can be moare equalle."

Later, = became associated with some algebraic properties, namely reflexivity, symmetry, transitivity, and substitutivity. Today, it is defined by those properties, not as an abbreviation for some words. Someone might say that Alice and Bob are equal tennis players because they have played each other 10 times, and each has won 5 matches. They might similarly say that Bob and Carol are equal tennis players because they too have played each other 10 times, and each has won 5 matches. But this kind of equality is not transitive. As it happens, Alice and Carol are unequal tennis players: they have played each other 10 times, and Alice has won 8 matches. Because of the lack of transitivity, no mathematician today would use $=$ for tennis equality.

In the notation commonly used for small sets, such as $\{1,3,7\}$, the comma was introduced as just punctuation, not as a mathematical operator. As soon as the notation is introduced, we must say that the order in which elements are written is irrelevant so that $\{1,2\}=\{2,1\}$; the way to say that formally is $A, B=B, A$ (comma is commutative). We must also say that repetitions of elements are irrelevant so that $\{3,3\}=\{3\}$; the way to say that formally is $A, A=A$ (comma is idempotent). And we should say that comma is associative $A,(B, C)=(A, B), C$ so that parentheses are unnecessary. Evidently the comma can be seen as a mathematical operator with algebraic properties that aggregates elements into a structure that is simpler, more primitive, than sets; let us call them bunches. Even the curly braces can be seen as an operator that applies to a bunch and makes a set; its inverse $\sim$ applies to a set and makes a bunch: $\sim\{1,2\}=1,2$.

When a child first learns about sets, there is often an initial hurdle: that a set with one element is not the same as the element. It would be easier to present a set as packaging: a package with an apple in it is obviously not the same as the apple. Just as $\{1\}$ and 1 differ, so $\{1,2\}$ and 1,2 differ. Bunch theory tells us about aggregation; set theory tells us about packaging. The two are independent.

Apart from being cute, are bunches useful? The subject of functional programming has suffered from an inability to express nondeterminism conveniently. To say something about a value, but not pin it down completely, one can express the set of possible values. Unfortunately, sets do not reduce properly to the deterministic case; in this context it is again a problem that a set containing one element is not equal to the element. What is wanted is bunches. One can always regard a bunch as a "nondeterministic value". Bunches can also be used as a "type theory" with the advantage that it is unnecessary to duplicate the operators of the value space at the type level because the two are unified. And finally, the easiest way to present sets is via bunches. For details see [13] [14]. Formalization of the lowly comma leads to a beautiful and useful algebra.

We have just seen two examples of formalization, one from the past and one from the future. Now here's an example of a formalization gone astray: functions defined as sets of ordered pairs. This way of defining functions is part of the very interesting demonstration that all of mathematics can be based on sets. The demonstration requires us to make a set-model of functions, and numbers, and everything else. For example, the natural numbers can be equated to sets, with no inconsistency, as follows: 


$$
\begin{aligned}
& 0=\varnothing \quad \text { (the empty set) } \\
& n+1=n \cup\{n\}
\end{aligned}
$$

So, for example, $3=\{\varnothing,\{\varnothing\},\{\varnothing,\{\varnothing\}\}\}$. Few people would say that 3 really is the set $\{\varnothing,\{\varnothing\},\{\varnothing,\{\varnothing\}\}\}$; the set-model of natural numbers was constructed by John von Neumann just to serve this one demonstration. Numbers are best formalized, not by building a set-model, but by an algebra showing how they participate in arithmetic operations. Similarly, functions are best formalized by showing the laws of application and function composition (in general, set union and intersection are not useful ways of combining functions). But the set-model of functions has somehow taken root in the current mathematical culture; many people (and textbooks) say that a function really is a set of ordered pairs. A useful formalization is not one that answers the question "what is it?", but one that answers the question "how do we use it?".

I write a function, or local scope, according to the following example:

$$
\langle n: n a t \rightarrow n+1\rangle
$$

This is essentially a "lambda-expression"[6], although Church did not use angle brackets and arrow. He borrowed a "hat" notation from Whitehead and Russell, but for typesetting convenience moved the hat down in front; the most similar available character in the typesetter's tray was $\lambda$; thus the lambda calculus was born [22]. Following van de Snepscheut [23], I use angle brackets to delimit the scope of the variable. I use an arrow to facilitate the unification of functions with function spaces, which I do not discuss in this paper (see [14]). Next, I want to get rid of the idea that all possible variables (infinitely many of them) already "exist", and that the function notation "binds" a variable, and any variable that is not bound remains "free". I prefer the programmer's terminology of "local" and "nonlocal" variables. Variables do not automatically "exist"; they are introduced (rather than bound) with a limited scope by the function notation.

Two notations that have not yet made the transition from informal beginning to formal, calculational tool are the quantifiers $\forall$ and $\exists$. For most mathematicians today they remain abbreviations for the words "for all" and "there exists", and their meaning is just whatever can be understood from those words. The word "all" sounds clear and unambiguous, but there is debate as to whether so-called "undefined" range elements, or other "nonstandard" elements, are included. Existence is even more contentious, as can be seen from the debate between classical and constructive mathematicians. Only a formal definition, equivalent to an automated theorem prover, is clear and unambiguous. Only a formal definition gives us calculation.

\section{Quantifiers}

There are several commonly used notations that introduce a local (bound, dummy) variable. For examples,

$$
\sum_{x=0}^{\infty} f(x) \quad \quad \quad \int_{a}^{b} f(x) d x \quad \forall x: D \cdot P(x) \quad\{f(x) \mid x \in D\}
$$

The introduction of the local variable and its domain are exactly the job of the function notation, so all expressions requiring a local variable can be uniformly expressed as an operator applied to a function. If the body of a function is a number expression, then we can apply + to obtain the sum of the function results. For example,

$$
+\left\langle n: \text { nat } \rightarrow 1 / 2^{n}\right\rangle
$$

There is no syntactic ambiguity caused by this use of + , so no need to employ another symbol $\Sigma$ for addition. We can apply any associative symmetric operator, such as 


$$
\begin{aligned}
& \times\left\langle n: \text { nat } \rightarrow 1 / 2^{n}\right\rangle \\
& \downarrow\langle n: \text { nat } \rightarrow n>5\rangle \\
& \uparrow\langle n: \text { nat } \rightarrow n>5\rangle
\end{aligned}
$$

The minimum operator $\downarrow$ replaces "for all", and the maximum operator $\uparrow$ replaces "there exists". By applying $=$ and $\neq$ to functions we obtain the two independent parity operators. Set comprehension and integrals can be treated this same way.

If function $f$ has domain $D$, then $f=\langle x: D \rightarrow f x\rangle$, so quantifications traditionally written

$$
\sum_{x: D} f(x) \quad \forall x: D \cdot P(x)
$$

which we can now write as

$$
+\langle x: D \rightarrow f x\rangle \quad \downarrow\langle x: D \rightarrow P x\rangle
$$

can be written even more succinctly as

$$
+f \quad \downarrow P
$$

Using adjacency for composition, deMorgan's laws

become

$$
\neg(\forall x: D \cdot P x) \equiv(\exists x: D \cdot \neg P x) \quad \neg(\exists x: D \cdot P x) \equiv(\forall x: D \cdot \neg P x)
$$

$$
-\downarrow P=\uparrow-P \quad-\uparrow P=\downarrow-P
$$

The Specialization and Generalization laws say that if $y$ is an element of $D$,

$$
(\forall x: D \cdot P x) \Rightarrow P y \quad P y \Rightarrow(\exists x: D \cdot P x)
$$

They now become

$$
\downarrow P \leq P y \quad P y \leq \uparrow P
$$

which say that the minimum item is less than or equal to any item, and any item is less than or equal to the maximum item. These laws hold for all numbers, not just for the booleans.

Given a function $f$, we can subtract $y$ from each function value, and then find the minimum, or we can save a lot of subtractions by finding the minimum function value and then subtracting $y$ from that.

$$
\begin{array}{ll} 
& \downarrow\langle x \rightarrow f x-y\rangle \\
= & \downarrow\langle x \rightarrow f x\rangle-y \\
= & \downarrow f-y
\end{array}
$$

The same calculation can be performed if - is replaced by $\geq$.

$$
\begin{array}{lll} 
& \downarrow\langle x \rightarrow f x \geq y\rangle & \text { factor out } \geq y \\
= & \downarrow\langle x \rightarrow f x\rangle \geq y & \\
= & \downarrow f \geq y &
\end{array}
$$

The traditional reading of the top line is: for all $x, f$ of $x$ is at least $y$. But the middle line is read: the minimum function value is at least $y$. It is the same $\downarrow$ operator on both lines, but traditionally it is pronounced "for all" on one line and "minimum" on the other. Or maybe $f x$ and $y$ are both boolean, and $\geq$ is reverse implication, in which case the top line would traditionally be read: for all $x,(f$ of $x$ is implied by $y)$; and the middle line would traditionally be read: (for all $x, f$ of $x$ ) is implied by $y$. No matter whether $f$ is a numeric or boolean function, no matter whether $\geq$ is a numeric or boolean comparison, the law is the same.

If we factor from the other side of the - sign, we have to change minimum to maximum: 


$$
\begin{array}{ll} 
& \downarrow\langle x \rightarrow y-f x\rangle \\
= & y-\uparrow\langle x \rightarrow f x\rangle \\
= & y-\uparrow f
\end{array}
$$

factor out $y-$

Once again replacing - with $\geq$, we have the same calculation.

$$
\begin{array}{ll} 
& \downarrow\langle x \rightarrow y \geq f x\rangle \\
= & y \geq \uparrow\langle x \rightarrow f x\rangle \\
= & y \geq \uparrow f
\end{array}
$$

factor out $y \geq$

This has several traditional readings depending on what's boolean and what's numeric. But it is just one law. Unified algebra gives us many other factoring/distribution laws just like these (see [14]).

The goal is to create an algebra that's easy to learn and easy to use. That goal is not always consistent with traditional mathematical terminology and symbology. The reader is cautioned against matching the algebra directly against their own familiar terms and symbols. Although I have been using the words "minimum" and "maximum" for $\downarrow$ and $\uparrow$, the words "greatest lower bound" and "least upper bound", or "infimum" and "supremum", may be more traditional in some contexts. For example,

$$
\downarrow\langle n: n a t \rightarrow 1 / n\rangle=0
$$

Even more caution must be used with the words "all" and "exists". Intuition about existence in mathematics (like intuition about anything else) depends on what you have learned. We tend to believe that what we have learned is true. But mathematical truth is constructed, and we must be open to the possibility of constructing it differently. Unlearning can be more difficult than learning.

\section{Quantifier Examples}

Is $(\exists x \cdot P x) \Rightarrow(\forall y \cdot Q y)$ equivalent to $\forall x \cdot \forall y \cdot(P x \Rightarrow Q y)$ ? Even experienced logicians don't find it obvious. To see if they are equivalent, those who reason informally say things like "suppose some $x$ has property $P$ ", and "suppose all $y$ have property $Q$ ". They are led into case analyses by treating $\forall$ and $\exists$ as abbreviations for "for all" and "there exists" (as they originally were). Of the very few who reason formally, most don't know many laws; perhaps they start by getting rid of the implications in favor of negation and disjunction, then use deMorgan's laws. Let me rewrite the question in the new notations.

$$
(\uparrow P \leq \downarrow Q)=\downarrow\langle x \rightarrow \downarrow\langle y \rightarrow P x \leq Q y\rangle\rangle
$$

We might read the left side as saying that the maximum $P$ is less than or equal to the minimum $Q$, and we might read the right side as saying that all $P$ are less than or equal to all $Q$. Informal readings can be misleading, and we should never attach our understanding to an informal reading, but sometimes we can get inspiration from it. In this case, the reading sounds reasonable enough to suggest we might prove it, and not just for booleans, but for all numbers. Leaving the non-null domains implicit, here's the two-step proof:

$$
\begin{array}{ll} 
& \downarrow\langle x \rightarrow \downarrow\langle y \rightarrow P x \leq Q y\rangle\rangle \\
= & \downarrow\langle x \rightarrow P x \leq \downarrow Q\rangle \\
= & \uparrow P \leq \downarrow Q
\end{array}
$$

factor out $P x \leq$

factor out $\leq \downarrow Q$

Let $L$ be a nonempty list (a function whose domain is an initial segment of the naturals). $+L$ is its sum, and $\uparrow L$ is its maximum; let $\# L$ be its length. We can say that the average item in the list is less than or equal to the maximum item as follows (leaving the domain implicit). 


$$
\begin{array}{ll} 
& +L / \# L \leq \uparrow L \\
\leq \quad & (+L / \# L>1) \leq(\uparrow L>1) \\
=\quad & (+L>\# L) \leq \uparrow\langle i \rightarrow L i>1\rangle
\end{array}
$$

now apply $>1$ to both sides of the inequality multiply by $\# L$; distribute $>1$

The bottom line is the "pigeon-hole principle"; it says that if the total number of things is greater than the number of places to put them, then some place has more than one thing in it. Notice what has happened: we read $\uparrow$ as "maximum" on the top line, and as "some" on the bottom line; we read $\leq$ as "less than or equal to" on the top line, and as "if then" on the bottom line.

Here is a further illustration of the benefits of unified algebra. Let $f$ be a function from the naturals to the reals. If $f$ is nondecreasing, then $f 0$ is its minimum. Traditionally, this might be written (leaving the domain implicit) as

$$
(\forall n \cdot f n \leq f(n+1)) \Rightarrow(f 0=\operatorname{MIN}\{f n \mid 0 \leq n<\infty\})
$$

Rewriting this in the new notation, and weakening it to say that $f 0$ is less than or equal to the minimum, we get

$$
\downarrow\langle n \rightarrow f n \leq f(n+1)\rangle \leq(f 0 \leq \downarrow f)
$$

Now we apply the portation law, which says that for boolean $a$ and any $b$ and $c$,

to obtain

$$
(a \leq(b \leq c))=(a \downarrow b \leq c)
$$

$$
f 0 \downarrow \downarrow\langle n \rightarrow f n \leq f(n+1)\rangle \leq \downarrow f
$$

If $f$ happens to have a boolean range, this is induction, more traditionally written

$$
f 0 \wedge(\forall n \cdot f n \Rightarrow f(n+1)) \Rightarrow(\forall n \cdot f n)
$$

Thus we see induction as a special case of a more general law saying that the first item in a nondecreasing sequence is its minimum.

\section{Probability}

The seminal work [4] by Boole on boolean algebra refers to both logic and probability. The standard theory of probability assigns 0 to an event that cannot happen, $1 / 2$ to an event that is equally likely to happen or not happen, and 1 to an event that is certain to happen. In a set of events in which exactly one event must happen, the probabilities sum to 1 . The integral of a probability distribution must be 1 .

Perhaps there is another way to develop probability theory based on unified algebra. Perhaps an event that cannot happen has probability $\perp$, an event that is equally likely to happen or not happen has probability 0 , and an event that is certain to happen has probability $T$. In a set of events in which exactly one event must happen, the average probability is 0 . The integral of a probability distribution must be 0 . Perhaps the new probability space is related to the logarithm of the old space; essentially, probabilities are replaced by information content. My hope is that the complicated formulas for distributions in the standard theory can be simplified by transforming the space of probabilities.

\section{Metalogic}

In the study of logic, at or near the beginning, logicians present the symbol $\vdash$ to represent theoremhood. I ask you to put yourself in the place of a beginning student. This symbol is applied to a boolean expression just like the boolean operators; but we know all the boolean operators and this isn't one of them. It sometimes has a left operand as well as a right operand, and then the explanation makes it seem just like implication. To say that it is a "meta-operator" just labels it, and doesn't explain it. Saying that it applies to the form, rather than the meaning, is 
confusing too, since the entire point of the algebra is to enable us to work with the form and ignore the meaning. The distinction between metanotations and the object notations is not easily seen.

To make things worse, there are different levels of meta-operators. Proof rules are sometimes presented using a horizontal line, which is yet another level of implication. Consider, for example, the Modus Ponens proof rule, which uses all three kinds of implication:

$$
\frac{A \vdash x, B \vdash x \Rightarrow y}{A, B \vdash y}
$$

Rewriting comma as conjunction, and turnstile and line as implication, we get a tautology:

$$
(A \leq x) \downarrow(B \leq(x \leq y)) \leq(A \downarrow B \leq y)
$$

Rewriting any proof rule this way gives a tautology (if $\vdash$ has nothing to its left, use $T$ ). Rewriting any tautology whose main connective is implication gives a valid proof rule. It is hard to see the difference between the meta-operators and the object-level operators because there is no formal difference! The proof rules are used to explain how to use the boolean expressions; natural language is used to explain how to use the proof rules. For beginners (and others) it would be better to skip the meta-notations altogether and just use natural language to explain how to use the boolean expressions.

At a more advanced level, when we want a formalism to study formalisms, we will need an operator that applies to the form of an expression. For that purpose, we do not need any new kind or level of operator. Rather, we need to do exactly what Gödel did when he encoded expressions, but we can use a better encoding. We need to do exactly what programmers do: distinguish program from data. One person's program may be a compiler writer's data, but when it is data, it is a character string. The character string " $a \uparrow-a$ " can be used as a code for the expression $a \uparrow-a$. We apply $\vdash$ to character strings so that $\vdash s$ is a theorem when the boolean expression represented by string $s$ is a theorem.

We have a name, "theorem", for a boolean expression that can be simplified to $T$, and an operator, $\vdash$, whose purpose is to identify theorems. Strangely, logicians have not introduced a name, say "antitheorem", for a boolean expression that can be simplified to $\perp$, and no operator such as $\dashv$, whose purpose is to identify antitheorems. Perhaps that's because "antitheorem" just means "negation of a theorem" in those logics having negation and an appropriate proof rule. But we bother to name both booleans, even though one is just the negation of the other.

I propose that logicians can improve metalogic by taking another lesson from programming. Instead of $\vdash$ and $\dashv$, we need only one operator to serve both purposes. It is called an interpreter. I want $I s$ to be a theorem if and only if $s$ represents a theorem, and an antitheorem if and only if $s$ represents an antitheorem. It is related to $\vdash$ and $\dashv$ by the two implications

$$
\vdash s \leq \mathrm{I} s \leq-\dashv s
$$

In fact, if we have defined $\vdash$ and $\dashv$, those implications define $I$. But I want $I$ to replace $\vdash$ and $\dashv$ so I shall instead define it by showing how it applies to every form of boolean expression. Here is the beginning of its definition.

$$
\begin{aligned}
& I " T \text { " }=\mathrm{T} \\
& I " \perp "=\perp \\
& I("-", s)=-I s \\
& \mathrm{I}(s “ \downarrow " t)=\mathrm{I} s \downarrow \mathrm{I} t \\
& \mathrm{I}(s " \uparrow " t)=\mathrm{I} s \uparrow \mathrm{I} t
\end{aligned}
$$

And so on. For example, 


$$
\text { I “- } \top \downarrow \perp \text { ” = - } \downarrow \downarrow \perp=\perp
$$

In a vague sense $I$ acts as the inverse of quotation marks; it "unquotes" its operand. That is what an interpreter does: it turns passive data into active program. It is a familiar fact to programmers that we can write an interpreter for a language in that same language, and that is just what I am doing here. Interpreting (unquoting) is exactly what logicians call Tarskian semantics. In summary, an interpreter is a better version of $\vdash$, and strings make metalevel operators unnecessary.

Using I, the famous Gödel incompleteness proof is just 3 lines. Suppose that every boolean expression is either a theorem or an antitheorem (a complete logic), and define $Q$ by

Then

$$
Q=\text { " } \mathrm{I} Q "
$$

$$
\begin{array}{ll} 
& \mathrm{I} Q \\
= & \mathrm{I} \text { “ }-\mathrm{I} Q \\
= & -\mathrm{I} Q
\end{array}
$$

replace $Q$ with its equal I unquotes

which proves a boolean expression equal to its negation, showing the logic to be inconsistent. A logic in which we can define an interpreter, and in which we can replace an expression with its equal, must be inconsistent or incomplete. We choose consistency, and we choose to allow the replacement of an expression with its equal, so we are forced to give up the ability to define a complete interpreter; in particular, I cannot unquote " $-I Q$ " . For further details of this version of Gödel's incompleteness theorem, see [12].

You cannot learn a programming language by reading an interpreter for it written in that same language. And you cannot learn logic, or a logic, by reading an interpreter for it written in logic. Not only is it inscrutable to a novice, but also it may be subject to more than one interpretation. Logic is better presented as algebra [11]. We don't present number algebra with the aid of a metaoperator that applies to number expressions and results in their values, and we should not present boolean algebra that way. I think boolean algebra should be presented with a little natural language and a lot of laws, because laws don't use any metanotations.

\section{Terms of Honor}

My final comment concerns mathematical terminology intended to honor mathematicians. In some parts of mathematics it is standard: Lie algebra, Stone algebra, Cartesian product, Jordan decomposition, Cayley transform, Hilbert space, Banach space, Hausdorff space, Borel measure, Lebesgue integration, Fredholm index, Wedderburn's Theorem, and so on. It is well known that the person so honored is sometimes the wrong person; often it is only one of many who equally deserve to have their names attached to the idea. I suspect that sometimes the intention is not so much to honor a person as to use the person's prestige to lend respectability to an idea. Even when the intention is to honor, the effect is to obscure and make the mathematics forbidding and inaccessible. It may be argued that this is good, keeping the uninitiated from thinking they understand when they don't, but I reject that argument as elitist. I know what nand and nor are, but I forget which is the Scheffer stroke and which the Peirce arrow. To say that an operator is symmetric or commutative is much more descriptive and understandable than calling it Abelian. DeMorgan's laws would be better named duality laws. We who are used to the terms forget what a barrier they pose to beginners. 
The term "boolean algebra" honors George Boole. (It is popularly thought that the word "algebra" honors someone, but it comes from an Arabic word meaning "the reintegration and reunion of broken parts". In any case, the word is now standard, known by people everywhere.) The best way to honor George Boole is to make the algebra that he created [4] a well known and well used tool, and to do that we might have to remove his name from it, and give it a more descriptive and accessible name, like "binary algebra".

\section{Conclusions}

Logic has been well studied and is now well understood, but it is not well used. Programmers learn that logic is a foundation of programming, but they don't often use it to program. Mathematicians study about logic, but they don't often use it in their proofs. Logic is a tool, like a knife. People have looked at it from every angle; they've described how it works at great length; now it's time to pick it up and use it. To use logic well, one must learn it early, and practice a lot. Fancy versions of logic, such as modal logic and metalogic, can be left to university study, but there is a simple basic algebra that can be taught early and used widely.

Number algebra is used by scientists and engineers everywhere. It is used by economists and architects. It is taught first to 6-year olds, without a metanotation, very concretely as addition and subtraction of numbers. Then variables and equations are introduced, and always the applications are emphasized. As a result of that early and long education, scientists and engineers and mathematicians are comfortable with it. Boolean algebra can be equally useful if it is taught the same way. At present, it is not in a good state for presentation to a wide audience. We need to simplify the terminology, get rid of the metanotations, adopt the view that proof is calculation, choose some good symbols, detach it from its dominant application in which the boolean values represent true and false statements, and explain it as algebra.

There is a small advantage to choosing uniquely boolean symbols: we can give them a precedence after the arithmetic operators, which reduces the need for parentheses. On the other hand, there is a large advantage to uniting boolean and number symbols in the way I have suggested: the laws and solutions are familiar and can be interpreted either as booleans or numbers. In addition, by placing booleans in the same context as numbers, we move quickly away from debates about the meanings of operators. The fact that the booleans can be embedded in the extended integers just as smoothly as the integers are embedded in the rationals seems a compelling reason to do so.

Quantifiers can be simplified, made uniform, and generalized by treating them as operators on functions. We should stop speaking about "existence", and speak instead about the maximum of a function. Similarly, we should stop speaking about "all", and speak instead about the minimum of a function. We should stop trying to say what functions and other mathematical ideas are, and say instead how to write them and use them.

An interpreter serves the same purpose as the metalevel theoremhood operator with the added advantage that it gives antitheoremhood as well as theoremhood. And by applying it to strings, we don't need to introduce a separate metalevel of operators. Metalogic is an advanced topic, not a good introduction to boolean algebra for those who are new to the subject.

This paper has not presented a detailed proposal for a change to our primary and secondary mathematics curriculum, but it has presented the case for making a change, and several 
suggestions. The main suggestion is to unify boolean algebra with number algebra so that we can begin with the simplest algebra and move smoothly to the more complicated algebras, all using the same notations and in the same calculational framework.

\section{Acknowledgment}

Theo Norvell provided references [16] and [19]. Benet Devereux provided references [3] and [18]. Rutger M. Dijkstra, Wim Hesselink, and Jim Grundy corrected some errors and caused me to improve some explanations.

\section{References}

[0] Unfortunately, 500 year old algebra texts are hard to find. This is not a quotation, but my own creation. I think it is representative of the work of the time, such as [20]; see the second Appendix.

[1] L.E.Allen: "Symbolic Logic: a Razor-Edged Tool for Drafting and Interpreting Legal Documents", the Yale Law Journal v.66 p.833-879, 1957

[2] R.Back, J.Grundy, J.vonWright: "Structured Calculational Proof", Formal Aspects of Computing v.9 n.5 p.469-483, 1997

[3] J.M.Bochinsky: a History of Formal Logic second edition, translated and edited by Ivo Thomas, Chelsea Publishing, New York, 1970

[4] G.Boole: an Investigation of the Laws of Thought, on which are founded the Mathematical Theories of Logic and Probabilities, MacMillan, 1854, reprinted by Dover, 1973

[5] R.Boute: "Binary Algebra and Functional Predicate Calculus: a Practical Approach", University of Ghent, Belgium, 1999

[6] A.Church: "the Calculi of Lambda-Conversion", Annals of Mathematical Studies v.6, Princeton University Press 1941

[7] E.W.Dijkstra, C.S.Scholten: Predicate Calculus and Program Semantics, Springer-Verlag 1990

[8] R.L.Goodstein: Development of Mathematical Logic, Springer-Verlag 1971

[9] D.Gries: "Improving the Curriculum through the teaching of Calculation and Discrimination", Communications of the ACM v.34 n.3 p.45-55, 1991 March

[10] D.Gries, F.B.Schneider: a Logical Approach to Discrete Math, Springer-Verlag, 1993

[11] P.Halmos, S.Givant: Logic as Algebra, the Mathematical Association of America, 1998

[12] E.C.R.Hehner: "Beautifying Gödel", chapter 18 in Beauty is our Business, a birthday tribute to Edsger Dijkstra, Springer-Verlag, 1990; www.cs.toronto.edu/ hehner/God.pdf

[13] E.C.R.Hehner: a Practical Theory of Programming, Springer-Verlag 1993; second edition 2004, www.cs.toronto.edu/ hehner/aPToP

[14] E.C.R.Hehner: "Unified Algebra", www.cs.toronto.edu/ hehner/UA.pdf

[15] I.N.Herstein: Topics in Algebra p.323, Blaisdell 1964.

[16] R.H.Katz: Contemporary Logic Design, Benjamin Cummings 1994 p.10

[17] L.Lamport: "How to Write a Proof", American Mathematical Monthly v.102 n.7 p.600-608 1995 Aug.

[18] J.Lukasiewicz: "on the History of the Logic of Propositions", Selected Works, citing Sextus Empiricus in Adversus Mathematicos, circa 200

[19] C.Navarre: quoted in Barbara W. Tuchman: a Distant Mirrror: the Calamitous Fourteenth Century, Knopf 1978

[20] R.Recorde: the Whetstone of Witte, London 1557, reprinted by Da Capo Press Amsterdam 1969. 
[21] P.J.Robinson, J.Staples: "Formalizing a Hierarchical Structure of Practical Mathematical Reasoning", Journal of Logic and Computation 3(1):47-61, February 1993

[22] J.B.Rosser: "Highlights of the History of the Lambda-Calculus", Annals of the History of Computing v.6 n.4 p.337-349, 1984 October

[23] J.L.A.van de Snepscheut: What Computing is All About, Springer-Verlag, 1993

[24] J.M.Spivey: the Z Notation: a Reference Manual, Prentice-Hall, 1989 


\section{Appendix}

Wedderburn's Theorem: a finite division ring is a commutative field (verbatim from [15])

Proof: Let $D$ be a finite division ring and let $Z$ be its center. By induction we may assume that any division ring having fewer elements than $D$ is a commutative field.

We first remark that if $a, b \in D$ are such that $b^{t} a=a b^{t}$ but $b a \neq a b$ then $b^{t} \in Z$. For, consider $N\left(b^{t}\right)=\left\{x \in D \mid b^{t} x=x b^{t}\right\} . N\left(b^{t}\right)$ is a subdivision ring of $D$; if it were not $D$, by our induction hypothesis, it would be commutative. However, both $a$ and $b$ are in $N\left(b^{t}\right)$ and these do not commute; consequently, $N\left(b^{t}\right)$ is not commutative so must be all of $D$. Thus $b^{t} \in Z$.

Every nonzero element in $D$ has finite order, so some positive power of it falls in $Z$. Given $w \in D$ let the order of $w$ relative to $Z$ be the smallest positive integer $m(w)$ such that $w^{m(w)} \in Z$. Pick an element $a$ in $D$ but not in $Z$ having minimal possible order relative to $Z$, and let this order be $r$. We claim that $r$ is a prime number for if $r=p q$ with $1<p<r$ then $a^{p}$ is not in $Z$. Yet $\left(a^{p}\right)^{q}=a^{r} \in Z$, implying that $a^{p}$ has an order relative to $Z$ smaller than that of $a$.

By the corollary to Lemma 7.9 there is an $x \in D$ such that $x a x^{-1}=a^{i} \neq a$; thus $x^{2} a x^{-2}=x\left(x a x^{-1}\right) x^{-1}=x a^{i} x^{-1}=\left(x a x^{-1}\right)^{i}=\left(a^{i}\right)^{i}=a^{i 2}$. Similarly, we get $x^{r-1} a x^{-(r-1)}=a^{i(r-1)}$. However, $r$ is a prime number thus by the little Fermat theorem (corollary to Theorem $2 . \mathrm{a}$ ), $i^{r-1}=1+u r$, hence $a^{i(r-1)}=a^{1+u r}=a a^{u r}=\lambda a$ where $\lambda=a^{u r} \in Z$. Thus $x^{r-1} a=\lambda a z x^{r-1}$. Since $x \notin Z$, by the minimal nature of $r, x^{r-1}$ cannot be in $Z$. By the remark of the earlier paragraph since $x a \neq a x, x^{r-1} a \neq a x^{r-1}$ and so $\lambda \neq 1$. Let $b=x^{r-1}$; thus $b a b^{-1}=\lambda a$; consequently, $\lambda^{r} a^{r}=\left(b a b^{-1}\right)^{r}=b a^{r} b^{-1}=a^{r}$ since $a^{r} \in Z$. This relation forces $\lambda^{r}=1$.

We claim that if $y \in D$ then whenever $y^{r}=1$, then $y=\lambda^{i}$ for some $i$, for in the field $Z(y)$ there are at most $r$ roots of the polynomial $u r^{r}-1$; the elements $1, \lambda, \lambda^{2}, \ldots, \lambda^{r-1}$ in $Z$ are all distinct since $\lambda$ is of the prime order $r$ and they already account for $r$ roots of $u^{r}-1$ in $Z(y)$, in consequence of which $y=\lambda i$.

Since $\lambda^{r}=1, b^{r}=\lambda^{r} b^{r}=(\lambda b)^{r}=\left(a^{-1} b a\right)^{r}=a^{-1} b^{r} a$ from which we get $a b^{r}=b^{r} a$. Since $a$ commutes with $b^{r}$ but does not commute with $b$, by the remark made earlier, $b^{r}$ must be in $Z$. By Theorem 7.b the multiplicative group of nonzero elements of $Z$ is cyclic; let $\gamma \in Z$ be a generator. Thus $a^{r}=\gamma^{j}, b^{r}=\gamma^{k}$; if $j=s r$ then $a^{r=\gamma^{s r}}$; whence $\left(a / \gamma^{s}\right)^{r}=1$; this would imply that $a / \gamma^{s}=\lambda^{i}$, leading to $a \in Z$, contrary to $a \notin Z$. Hence, $r$ does not divide $j$; similarly $r$ does not divide $k$. Let $a_{1}=a^{k}$ and $b_{1}=b j$; a direct computation from $b a=\lambda a b$ leads to $a_{1} b_{1}=\mu b_{1} a_{1}$ where $\mu=\lambda-j k \in Z$. Since the prime number $r$ which is the order of $\lambda$ does not divide $j$ or $k, \lambda j k \neq 1$ whence $\mu \neq 1$. Note that $\mu^{r}=1$.

Let us see where we are. We have produced two elements $a_{1}, b_{1}$ such that:

(1) $a_{1}{ }^{r}=b_{1}{ }^{r}=\alpha \in Z$.

(2) $a_{1} b_{1}=\mu b_{1} a_{1}$ with $\mu \neq 1$ in $Z$.

(3) $\mu^{r}=1$.

We compute $\left(a_{1}^{-1} b_{1}\right)^{r} ;\left(a_{1}^{-1} b_{1}\right)^{2}=a_{1}^{-1} b_{1} a_{1}^{-1} b_{1}=a_{1}^{-1}\left(b_{1} a_{1}^{-1}\right) b_{1}=a_{1}^{-1}\left(\mu a_{1}^{-1} b_{1}\right) b_{1}=\mu a_{1}^{-2} b_{1}^{2}$. If we compute $\left(a_{1}^{-1} b_{1}\right)^{3}$ we find it equal to $\mu^{1+2} a_{1}^{-3} b_{1}^{3}$. Continuing we obtain $\left(a_{1}^{-1} b_{1}\right)^{r}=\mu^{1+2+\ldots+(r-1)} a_{1}^{-r} b_{1}^{r}=\mu^{1+2+\ldots+(r-1)}=\mu^{r(r-1) / 2}$. If $r$ is an odd prime, since $\mu^{r}=1$, we get $\mu^{r(r-1) / 2}=1$, whence $\left(a_{1}^{-1} b_{1}\right)^{r}=1$. Being a solution of $y^{r}=1, a_{1}^{-1} b_{1}=\lambda^{i}$ so that $b_{1}=\lambda^{i} a_{1}$; but then $\mu b_{1} a_{1}=a_{1} b_{1}=b_{1} a_{1}$, contradicting $\mu \neq 1$. Thus if $r$ is an odd prime number, the theorem is proved.

We must now rule out the case $r=2$. In that special situation we have two elements $a_{1}, b_{1} \in D$ such that $a_{1}^{2}=b_{1}^{2}=\alpha \in Z, a_{1} b_{1}=\mu b_{1} a_{1}$ where $\mu^{2}=1$ and $\mu \neq 1$. Thus $\mu=-1$ and $a_{1} b_{1}=-b_{1} a_{1} \neq b_{1} a_{1}$; in consequence, the characteristic of $D$ is not 2 . By Lemma 7.7 we can find elements $\zeta, \eta \in Z$ such that $1+\xi^{2}-\alpha \eta^{2}=0$. Consider $\left(a_{1}+\zeta b_{1}+\eta a_{1} b_{1}\right)^{2}$; on computing this out we find that $\left(a_{1}+\zeta b_{1}+\eta a_{1} b_{1}\right)^{2}=\alpha\left(1+\zeta^{2}-\alpha \eta^{2}\right)=0$. Being in a division ring this yields that $a_{1}+\zeta b_{1}+\eta a_{1} b_{1}=0$; thus $0 \neq 2 a_{1}^{2}=a_{1}\left(a_{1}+\xi b_{1}+\eta a_{1} b_{1}\right)+\left(a_{1}+\xi b_{1}+\eta a_{1} b_{1}\right) a_{1}=0$. This contradiction finishes the proof and Wedderburn's theorem is established. 


\section{Appendix}

Four pages from R.Recorde: the Whetstone of Witte, London 1557, reprinted by Da Capo Press Amsterdam 1969.

\section{The Arte}

The rule of equation, common. ly called Algebers Rule.

Tbe rule of equation.

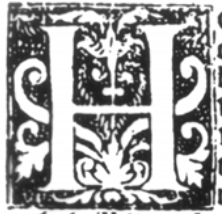

C therto baue $\mathbf{3}$ tauglote pou, the comnion foames of wojke, in noms jocrs Denominate, ขca biebe rules are vero alro in nöbers $A b$ fratte, $Q$ likes waics in Surde nomibers. Altbough the forme $\mathrm{s}$ of thefe wojkes be fcues ralle, in crbe linoc of noniber. $४ \mathrm{~B}$ ut nolw will $\mathbf{3}$ tcache vou that rule, that is the yzincipall in Coßike woojlies : ano foz whiche all the otber booe itrue.

Ih his hule is eallco the kisulc of Algeber, after the naute of the untentoure, as foinc incri rbintsc: o: by a jlanic of finsisular crecllencic, as otber inoge. 23 ut of his bfe it is rigbtlp calleo, the rulc of equation: bicaule that by equation of nombers, it Dorth oiffoluc boubtes full queftions: Ano bnfoloe intricate rioles. Ano this is the 0.oer of it.

Tbefomme of tbe rule of equation:

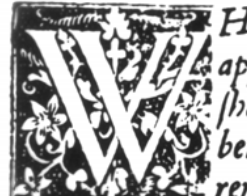

Hen any que/tion is propoinded, appertcinyng to this rule, you hall imagin a name for the nom. ar ber, that is to bec joughte, as you the rule of fallepofiomber, that you learned in ball rou procede accordion. And with that nomber you finde a Coßike nomber, equalle to that nom. ber, that the gueftion expreffeth, whic be you fhal reduce

$$
\text { of Coßike nombers. }
$$

reduce ener more to the leafte nombers. Anclithen diuide the nomber of the leffer denomination, by the nomber of the greatefte denomination, and the quotient douth aunfwerc to the queftion. Ex. cept thegreater denominatio, doe beare the /igne of fome rooted nöber. For then mift youextralt the roote of that quotiente, accordyng to that figne of denomination.

bisolat. st f(cmetb) that this rulc is all one, with the rulc of falle pofition: ano thercfo3c mighte fo bec sallco: (repng it taketh a falfe nóber, to wozke with al.

FGafter. IE bis rule Docth farrecrecll that otber. Givo Duostb not take a false nomber, but a true noun, berfoz bis pofation, as it fijall bee occlareo anon. खe berbp it mate bec thoughte, to bee a rulc of won: oc rfull inucntion, that teacheth a manuc at the firte wozoc, to name a true nomber, befoze be knolweth $\mathrm{rcs}$ folutelp, lobat be bath named.

visut bicaufe that nanic is common to manp nom: bers (although not in onc queftion)and tbercfoze the name is obfcure, till the looze doe octed it, 3 thinke this rule inight locll bee called, the rulc of oarke poff. tion, 0 ; of a raunge pofiticn: but not of falfe pofition.

Silo fo2 the mo2e cafic allo apte looze in this arte wec Dooc commonly namie that oarke pofition. I. 2 . gllo with it boc we luozke, as the quetion intcnoeth, till twe come to the equation.

2I his rule of equation, is oiutoed by fouic men, into Tbe partes Diucrfe partes. As namely Scbeubelius doocth make. 3. of tbe rule. rules of it. Ano in the feconoe rule, be putteth. 3. feuc, ralle cannós. ome otber men maake a greater nöber of oittintiös in this rule. 13 ut 3 intenoc(as 3 thinke brite $\mathrm{fO}_{2}$ this treatice, whiche maie ferue as farre ff. 


\section{The Arte}

as their fojlas boe ertentoc) to bittinate it onelp into tiwos partes. Wa bercel the firtte is, vbew on nowher is

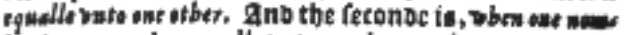
ber is compared as equalle buto. 2.0tber nombers.

alioates lwillyng pou to remerber, that pou rebuce pour nombers, to thetr lealte benominations, ano fmallefte fozmes, before pou pzotede anp farther.

and again, if pour equation be foche, that the greas tefle benomination Cofike, be foineo to anip parte of a compounoe nomber, gou ball tourne it fo, that the nomber of the greatelte figne alone, mate tanbe as cqualle to the refie.

Anb tbis is all that neabetb to be taugbte, concer: neng this woozke.

(1)olobeit, foz eatic alteratió of equations. I will pzo: pounbe a felwe erīples, bicaufe tbe ertraction of thetr rootes, maie the moze aptlp bec wjougbte. Ano to as, uoibe the tebioufe tepetition of thefe lwoozocs : is e, qualle to : 3 loill rette as 3 ooe ofteil in woozhe bre, paire of paralleles, 02 demoloe tines of onc lengtbe, thus: = , bicaufe noe. 2. thenges, can be moare equalle. Int notw marke thefe noinbers.

$$
\begin{aligned}
& \text { 1. } 14.2 .-15.9=-71.9 \text {. } \\
& \text { 2. } 20.2 e \text {. } 18.9-102.9 \text { - } \\
& \text { 3. 26.z-1-10\%e- } 9.3-107 e-1-21 ; .9 \text {. } \\
& \text { 4. 19.2e-1-192.9-107-1-1089-192e }
\end{aligned}
$$

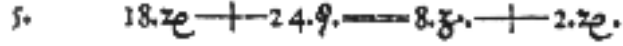

$$
\begin{aligned}
& \text { 6. } 34 z-12 \%-40 x e-1-4809-9 . z
\end{aligned}
$$
14.2 .

\section{of Cofsike nombers.}

14. 7 1. 5 . Yzut ff pou marke tbein loell, pou mate Ite one ucnominatio, on botbe fibes of the equation, fubicb ne. uer ougbt to frano. val berfoze abating the letter, that is. I s. 9. out of bothe the nombers, there bill remain. $14.70=-56.9$, that is, by rtbuation, $1=0=4.9$.

petyolar. 3 fet, pou abatt. 1 5.9.from tortm bothe. anb then are thei thatile sill, fepng thei wer cqualle befo;c. Actojbeng to the tbirbe common fentenet, in tbe pattbeloatc:

If jou abate cuen portiows, from sbynges tbat bee equallo, tbr partes tbat rewin Ball be equall aljo.

Mafter. pou boc betl reméber, the firte groun, bes of this artc. 1502 all fyzingett of thofe painciples Cetometricallit. Wu berfoze call to pour menbe litectuairs the ferendec common fetitenee, in thefame boolte, and then baue pou antotber reafon, whiche will belpe pou not anclp, in the otber foames of woodte bere, but ale fo beren oftcin in the pyactife of this arte.

acbolar. IIIbyt is this.

If you adde rqualle portions, to tbyoges tbat bee cqualle, thesfo amounterb of torm fhall be rgualle.

gaflec. Thefe twoo fentences boe inftruat pou that when poun fice on bothe the abes of the equation, as,

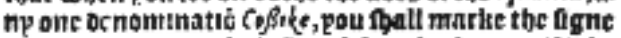
that is anucte b to the leficr of them botbe: anb if it be the figut of abstition.-- +- then fball pou abate tbat Icfiri nouluticr, froun bothe the partes of the equation.

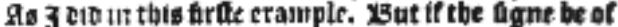
abateincute —-, then thall pou aboe that le terer nos ber, to botbe partes. Into fo flyall pou boc, till there be noc onc ornomination on bothe partes, but biuerfe and o tifindts.

5o tic fetonioc nomber will be.20.7m-1209 2

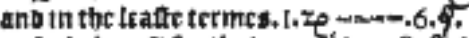

trbolal. 3 ite that qou cove. 18. 5\%. to bothe par, ff, t. tes 